Research Article

\title{
Cholinergic Urticaria: Clinical Presentation and Natural History in a Tropical Country
}

\author{
Chuda Rujitharanawong $(\mathbb{D}$, Papapit Tuchinda $(\mathbb{D}$, Leena Chularojanamontri $(\mathbb{D}$, \\ Nattacha Chanchaemsri $\mathbb{D}$, and Kanokvalai Kulthanan
}

Department of Dermatology, Faculty of Medicine Siriraj Hospital, Mahidol University, Bangkok, Thailand

Correspondence should be addressed to Kanokvalai Kulthanan; kanokvalai.kul@mahidol.ac.th

Received 3 March 2020; Accepted 6 May 2020; Published 27 May 2020

Academic Editor: Enrico Heffler

Copyright $(2020$ Chuda Rujitharanawong et al. This is an open access article distributed under the Creative Commons Attribution License, which permits unrestricted use, distribution, and reproduction in any medium, provided the original work is properly cited.

\begin{abstract}
Background. Cholinergic urticaria (CholU) is a subset of chronic inducible urticaria characterized by the recurrent pinpoint-sized wheals that are induced by exercising or increasing core body temperature. Currently, the data of CholU in tropical climate is still limited. Objective. To investigate the clinical features and natural course of CholU in a tropical country. Materials and Methods. This retrospective chart review study analyzed the data of CholU patients aged over 18 years who visited Siriraj Urticaria Clinic, Siriraj Hospital, Bangkok, Thailand, between January 2007 and September 2019. Demographic data, clinical presentations, and results of provocation tests and other laboratory investigations were evaluated and compared with other studies reported in temperate zones. Results. Sixteen out of 2,175 chronic urticaria patients $(0.7 \%)$ were diagnosed with CholU. The median age of CholU patients was $28.0 \pm 11.7$ years with male predominance $(56.3 \%)$. Three patients $(18.8 \%)$ had a history of atopy. Fifteen patients $(93.8 \%)$ were positive to the exercise provocation test. Nonsedating antihistamine drugs were a main treatment (73.8\%). Six patients $(37.5 \%)$ were in remission at the time of the study, with a mean duration 4.3 years. The Kaplan-Meier survival analysis demonstrated that $12.5 \%, 35.5 \%$, and $67.9 \%$ of patients would have disease remission within 1 year, 5 years, and 13 years, respectively. Conclusions. The prevalence of CholU differs in each geographic region and is found to be low in tropical countries with a median duration 4.3 years. The prevalence of atopy and anaphylaxis with CholU is also lower in tropical countries than in temperate.
\end{abstract}

\section{Introduction}

Cholinergic urticaria (CholU) is a variant of inducible urticaria induced by a rising core body temperature after exercising, sweating, eating of spicy foods, stress, or passive warming [1]. CholU mostly appears in pinpoint-sized and urticaria eruptions with severe itch localized to the trunk and limbs and usually disappears rapidly on its own within a few hours. However, most patients with CholU feel discomfort in the skin that disturbs their quality of life [2-4]. In severe patients, CholU may be related to exercise-induced anaphylaxis, a severe wheal-flare reaction with systemic involvement including difficult breathing, wheezing, or abdominal pain [5]. The reported prevalence of CholU in a general population varies from $0.023 \%$ to $11.2 \%$ in temperate zone countries [6-8]. CholU mostly affects patients with age onset in their second to third decade [9]. The pathogenesis of CholU is still unclear. Some CholU patients are occasionally associated with anhidrosis/hypohidrosis [10]. Acetylcholine is noted to induce both sweating and wheals after intradermal injection [11]. The sweating-associated urticaria has been proposed as the etiology of CholU. In addition, sweat allergy that is a type I hypersensitivity against a component of sweat is observed in patients with CholU [12].

The diagnosis of CholU is established by history and an appropriate provocation test that is suitable to the patient's age and general condition. Previously, both heavy exercise and the passive warming that can increase the core body temperature have been utilized to confirm the diagnosis of CholU. CholU is diagnosed if wheal-flare reaction develops instantly or within a few minutes after heavy exercise or passive warming and commonly disappears within 15-60 
minutes [13]. The intradermal injection of $100 \mu \mathrm{g}$ of methacholine in $0.1 \mathrm{ml}$ of saline solution was used as another test for CholU diagnosis in both clinical and research settings. Wheal-flare reaction that occurs within 1 minute of the injection indicates a positive reaction $[14,15]$. However, the positive methacholine skin test demonstrates a hypersensitivity to cholinergic mediators that is not specific to CholU [16]. Thus, it has become less popular. Nowadays, the European Academy of Allergology and Clinical Immunology Dermatology Section, the Global Allergy and Asthma European Network, the European Dermatology Forum, and the

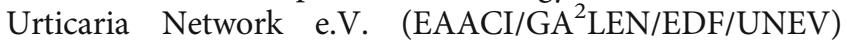
recommends 2 methods to investigate patients who are suspected CholU: (i) exercise machines: patients exercise by a bicycle trainer or treadmill for 30 minutes resulting in an increased pulse rate by 3 beats/minute every minute, and while a patient is testing, the doctor will monitor signs of CholU; (ii) a passive warming test: a person stays in a 42 degrees Celsius $\left({ }^{\circ} \mathrm{C}\right)$ bath, and a doctor monitors body temperature [1]. Patients continue the passive warming test for 15 minutes after body temperature increases $\geq 1^{\circ} \mathrm{C}$ over baseline. The positive result shows pinpoint-sized wheals during the test and ten minutes after ending the test [1].

The current EAACI/GA ${ }^{2} \mathrm{LEN} / \mathrm{EDF} / \mathrm{UNEV}$ consensus recommended the use of nonsedating histamine $\mathrm{H}_{1}$ receptor antagonist $\left(\mathrm{nsAH}_{1}\right)$ drugs as the first-line treatment, together with trigger avoidance as the best approach for CholU [1]. In refractory cases, updosing of $\mathrm{nsAH}_{1}$ and omalizumab (a recombinant humanized $\operatorname{IgG}_{1}$ monoclonal antibody that binds to $\operatorname{IgE}$ ) is recommended to improve the disease activity [1]. The addition of a $\mathrm{H}_{2}$ receptor antagonist $\left(\mathrm{AH}_{2}\right)$, propranolol ( $\beta_{2}$-adrenergic blocker), montelukast, and botulinum toxin injection has been reported as an effective treatment [1]. Furthermore, desensitization by routine exercise or treatment with autologous sweat in CholU patients with sweat allergy has been reported. Nevertheless, desensitization procedure should be conducted under physician observation due to risk of anaphylaxis [12, 17, 18]. Relating to the disease courses, the reported mean disease duration in patients who had disease remission was 4-7.5 years [1, 9, 19].

At present, there were only 2 studies of CholU in tropical countries. Godse et al. demonstrated the prevalence of CholU in Indian students by conducting a survey questionnaire [6]. Sánchez et al. reported physical and environmental factors which played a key role in CholU by questioning Colombian patients about triggers with exacerbation of CholU [19]. However, the mean duration of disease remission and remission rate had never been reported. Thus, the information of CholU in tropical climate is still limited. This study is aimed at investigating the clinical features and natural history of CholU in Thailand which is a tropical country.

\section{Materials and Methods}

This retrospective chart review study was designed to analyze preexisting data of CholU patients aged over 18 years who visited Siriraj Urticaria Clinic, Siriraj Hospital, Bangkok, Thailand, between January 2007 and September 2019. The study received the permission from the Siriraj Institutional
Review Board. Demographic data, clinical presentations, and results of provocation tests and other laboratory investigations were analyzed.

In this study, CholU was diagnosed by history of whealflare reaction and/or angioedema after exercise or increasing of the core body temperature and confirmed by provocation testing that matched the patient's age and general condition. The stationary bicycle was our routine provocation device. If patients were limited of physical activity, a passive warming test was performed by full bath at $42^{\circ} \mathrm{C}$ for up to 15 minutes until body core temperature increased more than $1^{\circ} \mathrm{C}[1]$. The positive result was the appearance of present tiny wheals that often localized on the limbs and trunk within 10 minutes [1]. All patients should discontinue any antihistamines at least for 1 week before the confirmation tests.

A remission of CholU was defined as an absence of wheal-flare reaction or angioedema regardless of increasing the body temperature and discontinuation of any urticaria treatment for at least 6 months. Of the remission cases, the disease duration was determined from the onset of symptoms to the remission date.

2.1. Statistical Analysis. In descriptive statistics, the demographic data, disease duration, and results of provocation testing and laboratory investigations were illustrated by mean, minimum, maximum, standard deviation, frequency, and percentages. The probability of remission among patients with CholU at each time point was determined by a Kaplan-Meier survival curve. The analysis of statistical data was assessed by the Statistical Package for the Social Sciences (SPSS) Statistics for Windows, version 18 (SPSS Inc., Chicago, IL, USA).

\section{Results}

Out of the 2,175 chronic urticaria patients who visited Siriraj Urticaria Clinic between January 2007 and September 2019, 178 patients $(8.2 \%)$ were diagnosed as inducible urticaria. Sixteen patients $(0.7 \%)$ with CholU diagnosis were enrolled in this study. The mean age was $28.0 \pm 11.7$ years (range 18-56 years). The majority were males (56.3\%). Table 1 shows the clinical characteristics of our CholU patients. Concomitant CSU was detected in 4 patients (25.0\%). One patient $(6.3 \%)$ has CholU coexisting with angioedema. No patients had symptoms of anaphylaxis. Three patients had positive atopic history (18.8\%), mostly allergic rhinitis $(n=2,12.5 \%)$. All patients were healthy with no known underlying disease.

All patients were investigated by the exercise provocation test. Fifteen patients (93.8\%) showed positive wheal and flare reaction. The only one patient with a negative exercise provocation test result had a confirmed history of wheal and angioedema after heavy exercise such as running and dancing.

Most of our CholU patients $(73.8 \%)$ were treated with nonsedating second-generation $\mathrm{H}_{1}$ antihistamine drugs $\left(\mathrm{nsAH}_{1}\right)$, i.e., fexofenadine (12 patients, 75\%), cetirizine (7 patients, $43.8 \%$ ), loratadine (6 patients, 37.5\%), desloratadine ( 2 patients, $12.5 \%$ ), bilastine ( 2 patients, $12.5 \%$ ), and 
TABLE 1: Characteristics and course of cholinergic urticaria in our 16 patients.

\begin{tabular}{|c|c|}
\hline Characteristics & Number (\%) \\
\hline \multicolumn{2}{|l|}{ Sex } \\
\hline Male & $9(56.3 \%)$ \\
\hline Female & $7(43.7 \%)$ \\
\hline Mean age of onset $(\mathrm{yr})$ & $28.0 \pm 11.7(15,56)$ \\
\hline Mean duration of cholinergic urticaria (yr) & 4.9 \\
\hline \multicolumn{2}{|l|}{ Associated other urticaria } \\
\hline Chronic spontaneous urticaria & $4(25.0 \%)$ \\
\hline Dermographism & $3(18.8 \%)$ \\
\hline Cold urticaria & $2(12.5 \%)$ \\
\hline Cold urticaria and dermographism & $1(6.3 \%)$ \\
\hline Angioedema & $1(6.3 \%)$ \\
\hline History of anaphylaxis & 0 \\
\hline Personal history of atopy & $3(18.8 \%)$ \\
\hline Allergic rhinitis & $2(12.5 \%)$ \\
\hline Asthma & $1(6.3 \%)$ \\
\hline Allergic conjunctivitis & 0 \\
\hline Atopic dermatitis & 0 \\
\hline \multicolumn{2}{|l|}{ Treatment ${ }^{\#}$} \\
\hline \multicolumn{2}{|l|}{$\mathrm{sAH}_{1}$} \\
\hline Hydroxyzine $(2)^{*}$ & $4(25 \%)$ \\
\hline \multicolumn{2}{|l|}{$\mathrm{nsAH}_{1}$} \\
\hline Cetirizine $(1-2)^{*}$ & $7(43.8 \%)$ \\
\hline Loratadine $(1-2)^{*}$ & $6(37.5 \%)$ \\
\hline Fexofenadine $(1-2)^{*}$ & $12(75 \%)$ \\
\hline Levocetirizine $(1)^{*}$ & $1(6.3 \%)$ \\
\hline Desloratadine $(1-2)^{*}$ & $2(12.5 \%)$ \\
\hline Bilastine $(2)^{*}$ & $2(12.5 \%)$ \\
\hline \multicolumn{2}{|l|}{$\mathrm{AH}_{2}$} \\
\hline Ranitidine $(2)^{*}$ & $4(25 \%)$ \\
\hline \multicolumn{2}{|l|}{ Remission (at the time of study) } \\
\hline Yes & $6(37.5 \%)$ \\
\hline No & $10(62.5 \%)$ \\
\hline $\begin{array}{l}\text { Mean disease duration } \\
\text { (in } 6 \text { cases with remission, yr) }\end{array}$ & $4.3 \pm 4.5(0.3,13.0)$ \\
\hline
\end{tabular}

The data were shown as mean \pm standard deviation (minimum, maximum) or number (\%). Abbreviations: $\mathrm{AH}_{2}: \mathrm{H}_{2}$ receptor antagonist; CSU: chronic spontaneous urticaria; $n \mathrm{nAH}_{1}$ : nonsedating $\mathrm{H}_{1}$ antihistamine; $\mathrm{sAH}_{1}$ : sedating $\mathrm{H}_{1}$ antihistamine; yr: year. ${ }^{\#}$ One patient might have one or more medications. ${ }^{*}$ The number of tablets per day.

levocetirizine (1 patient, 6.3\%). (Table 1) The dosage was ranged from standard up to 2-fold. Before the year 2014 when the EAACI/GA ${ }^{2} \mathrm{LEN} / \mathrm{EDF} / \mathrm{UNEV}$ consensus panel recommendations had been established, 4 patients had taken one or combination of $\mathrm{nsAH}_{1}$. Of sedating antihistamine drugs, hydroxyzine was the most common prescribed drug (4 patients, 25\%). Four patients (25\%) were treated with the combination of $\mathrm{nsAH}_{1}$ and $\mathrm{sAH}_{1}$. The recalcitrant patients to $\mathrm{AH}_{1}$ drugs needed other medications to control symptoms including ranitidine (4 patients, 25\%), cimetidine (1 patient,

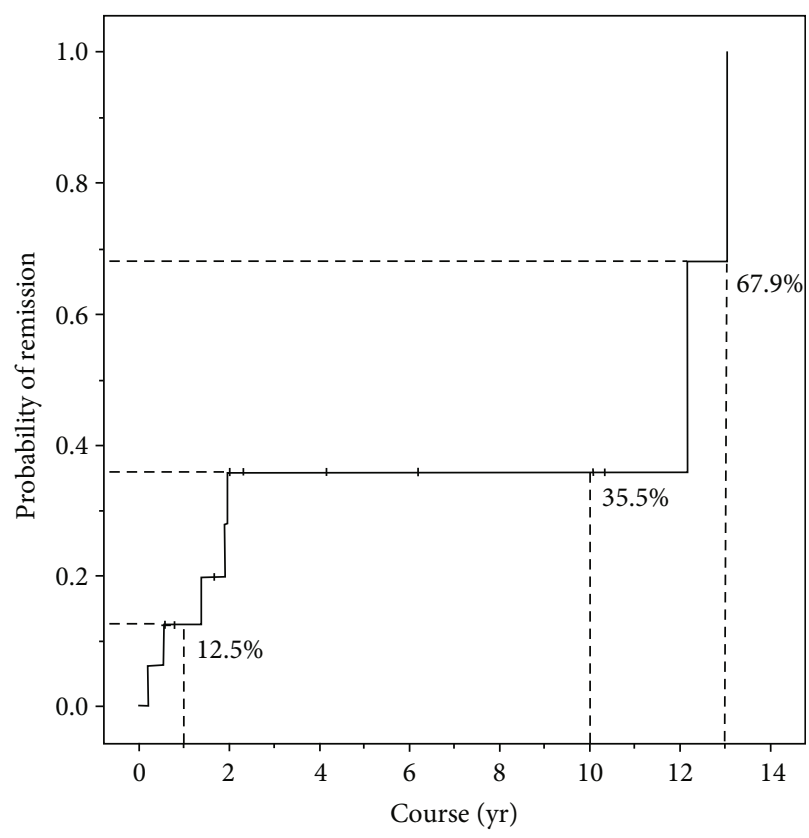

Figure 1: A Kaplan-Meier survival analysis (one minus survival function) demonstrated the remission during the first 2 years of CholU that was revealed in $5(31.3 \%)$ patients. In addition, 2 $(12.5 \%), 5(35.5 \%)$, and $6(67.9 \%)$ of patients were in remission after 1, 5, and 13 years, respectively, from the onset of CholU symptoms. The median duration when half of patients of CholU were in remission was 12.2 years. Abbreviations: CholU: cholinergic urticaria; yr: year.

$6.25 \%)$, propranolol (1 patient, $25 \%$ ), chloroquine (1 patient, $25 \%)$, and colchicine (1 patient, 25\%). Six patients (37.5\%) were in remission at the time of the study, with a mean disease duration $4.3 \pm 4.5$ years (range 0.3-13.0 years) as shown in Table 1. As stated in the Kaplan-Meier survival analysis, 5 patients $(31.3 \%)$ were in remission during the first 2 years of the disease. Figure 1 demonstrates that the remission within 1 year, 5 years, and 13 years was $12.5 \%, 35.5 \%$, and $67.9 \%$ of patients, respectively. After onset, the median duration before half of the patients were in remission was 12.2 years.

\section{Discussion}

In this study, the prevalence of CholU among chronic urticaria patients in a tropical country is $0.7 \%$. Our group previously reported the prevalence of CholU among chronic urticaria patients of $0.9 \%$ and $0.5 \%$ in the years 2007 and 2011, respectively [20,21]. The reported prevalence of CholU among general population in countries located in the tropical zones ranged from 0.023 to $11.2 \%$ [6-8]. Most studies including our study have shown the male predominance [6]. The mean age at symptom onset in our study was $28.0 \pm 11.7$ years that is consistent with the data reported in temperate zones [9]. Ten patients (62.5\%) had concomitantly other types of urticaria, mostly CSU. One patient had a combination of CholU, CSU, and dermographism.

Table 2 presents the information of CholU obtained in 3 temperate countries and 2 tropical countries compared with our study $[2,6-9,19]$. Although Thailand is located in the 


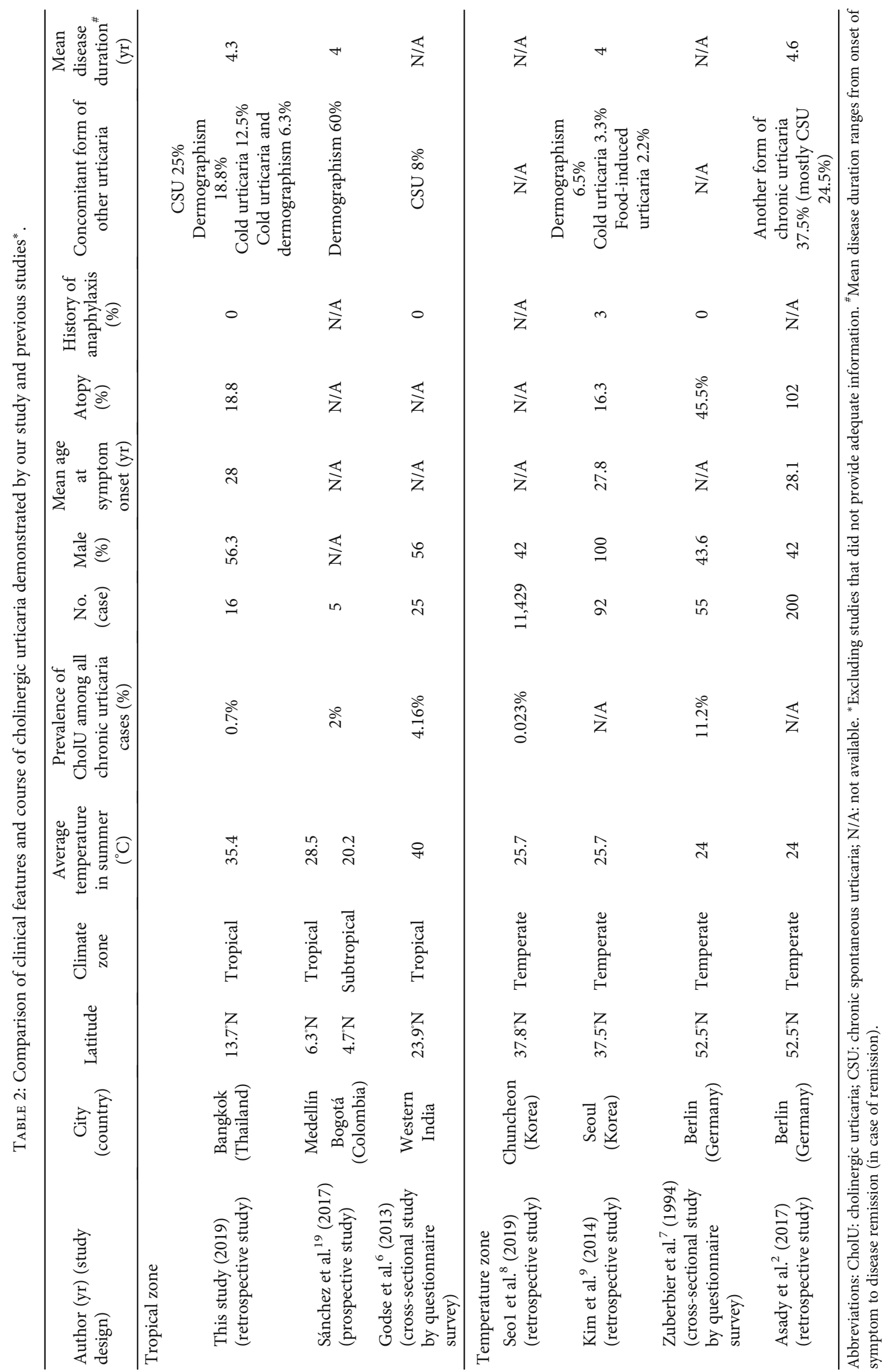


tropical zone and the latitude here seems to induce CholU, the prevalence of CholU is lower than in other temperate countries. We proposed that patients in the tropical zone are used to be in hot environment and the severity of CholU may be mild. Some patients misunderstand that the whealflare reaction is caused by sweating or irritation due to dust and do not see the doctor. Thus, the prevalence of CholU may be underdiagnosed in the tropical zone. Duke proposed that changes in temperature might be an associated factor in temperature-related urticaria, i.e., cold urticaria, rather than constant temperature [22]. Perhaps, the prevalence of CholU is less frequent in tropical countries due to less change in hot temperature. However, the supporting knowledge is still deficit to support that changes in temperature are likely to induce temperature-related urticaria (cold urticaria, heat urticaria, and CholU).

In this study, 3 patients (18.8\%) had positive histories of atopy, i.e., allergic rhinitis (2 patients, $12.5 \%)$ and asthma (1 patient, 6.3\%). In 2012, Vichyanond et al. reported the prevalence of allergic rhinitis and asthma in healthy subjects (26.3\% and $8.8 \%$, respectively) [23]. Nowadays, the prevalence of atopy trends to increase throughout the world [24]. These data implied that the prevalence of atopy was not raised in CholU patients in our study. Contrary to the studies of temperate countries, Zuberbier et al. reported the high prevalence of atopy in German patients with CholU (45.5\%) to be statistically significant demonstrated when compared with control persons (30.8\%) $(p<0.05)$ [7].

None of patients in our study had any systemic involvement or history of anaphylaxis. The result was consistent with the study of Godse et al. that no Indian patient presented with exercise-induced anaphylaxis [6]. To compare with temperate countries, 3 Korean patients (3.3\%) with chest tightness were reported by Kim et al. [9]. Respectively, CholU-induced anaphylaxis might be lesser noticed in tropical countries than in temperate countries.

Before 2016, the guideline treatment of physical urticaria was not established. Grattan et al. proposed that patients who failed with single dosage nsAH $\mathrm{H}_{1}$ could try at least $2 \mathrm{nsAH}_{1}$ together to control chronic urticaria [25]. Alsamarai et al. proposed that the combination of $\mathrm{AH}_{1}$ and $\mathrm{AH}_{2}$ was an effective treatment of CholU with complete control symptoms and a low relapsing rate [26]. Almost all patients in this study (15 patients) were treated by the combination of $\mathrm{AH}_{1}$ (mostly $\left.n s A H_{1}\right)$ and other medications including other nsAH, $\mathrm{sAH}_{1}$, $\mathrm{AH}_{2}$, and propranolol to control their disease symptom. At present, the current EAACI/GA ${ }^{2} \mathrm{LEN} / \mathrm{EDF} / \mathrm{UNEV}$ consensus recommendations for CholU management suggest $\mathrm{nsAH}_{1}$ as the first choice of treatment and trigger factor avoidance [1]. If standard dosing is insufficient to control symptoms, updosing of nsAH1 up to 4-fold is recommended. In recalcitrant patients, omalizumab is recommended as the second-line treatment to add with $\mathrm{AH}_{1}[1,27]$. Currently, novel biologics are being investigated as therapeutic options for CholU [28]. Ligelizumab, a humanized $\mathrm{IgG}_{1}$ monoclonal antibody, attaches to the $\mathrm{C} \varepsilon 3$ domain of $\operatorname{IgE}$ to inhibit interaction with the FCeRI on the surface of mast cells and basophils that is similar to omalizumab [29]. Quilizumab, a humanized $\operatorname{IgG}$ that binds the M1 prime segment of membrane-expressed IgE, has been demonstrated to decrease IgE levels in human subjects [30]. At present, both of them have been investigated in randomized controlled studies evaluating patients with refractory chronic inducible urticaria including CholU.

The Kaplan-Meier survival analysis demonstrated that the remission rate within 1 year, 5 years, and 13 years was $12.5 \%, 35.5 \%$, and $67.9 \%$ of our CholU patients, respectively. After onset, the median duration before half of the patients were in remission was 12.2 years. Limitations of our study were a limited number of patients with CholU and a retrospective study in which some data was missing. Further studies are needed to investigate rates of remission of CholU in other populations.

\section{Conclusion}

The prevalence of CholU is different in each geographic region, and the prevalence of CholU was found to be low in a tropical country with the median duration of CholU 4.3 years. The prevalence of atopy and anaphylaxis in patients with CholU in tropical countries is lower than that in temperate countries; however, other factors are indistinguishable between the tropical and temperate zones.

\section{Data Availability}

All research data and supporting information are included within the article.

\section{Conflicts of Interest}

All authors declare that there is no conflict of interest regarding the publication of this paper.

\section{Acknowledgments}

The authors gratefully acknowledge Orawan Supapueng, $\mathrm{PhD}$, of the Division of Clinical Epidemiology, Department of Research and Development, Faculty of Medicine Siriraj Hospital, for assistance with statistical analysis.

\section{References}

[1] M. Magerl, S. Altrichter, E. Borzova et al., "The definition, diagnostic testing, and management of chronic inducible urticarias - the EAACI/GA2LEN/EDF/UNEV consensus recommendations 2016 update and revision," Allergy, vol. 71, no. 6, pp. 780-802, 2016.

[2] A. Asady, J. Ruft, A. Ellrich, T. Hawro, M. Maurer, and S. Altrichter, "Cholinergic urticaria patients of different age groups have distinct features," Clinical \& Experimental Allergy, vol. 47, no. 12, pp. 1609-1614, 2017.

[3] M. Hatakeyama, A. Fukunaga, K. Washio et al., "Addition of lafutidine can improve disease activity and lead to better quality of life in refractory cholinergic urticaria unresponsive to histamine H1 antagonists," Journal of Dermatological Science, vol. 82, no. 2, pp. 137-139, 2016. 
[4] Y. Sawada, M. Nakamura, T. Bito et al., "Cholinergic urticaria: studies on the muscarinic cholinergic receptor M3 in anhidrotic and hypohidrotic skin," The Journal of Investigative Dermatology, vol. 130, no. 11, pp. 2683-2686, 2010.

[5] P. Vadas, A. Sinilaite, and M. Chaim, "Cholinergic urticaria with anaphylaxis: an underrecognized clinical entity," The Journal of Allergy and Clinical Immunology: In Practice, vol. 4, no. 2, pp. 284-291, 2016.

[6] K. Godse, N. Nadkarni, S. Patil, and S. Farooqui, "Prevalence of cholinergic urticaria in Indian adults," Indian Dermatology Online Journal, vol. 4, no. 1, pp. 62-63, 2013.

[7] T. Zuberbier, C. Althaus, S. Chantraine-Hess, and B. M. Czarnetzki, "Prevalence of cholinergic urticaria in young adults," Journal of the American Academy of Dermatology, vol. 31, no. 6, pp. 978-981, 1994.

[8] J.-H. Seo and J.-W. Kwon, "Epidemiology of urticaria including physical urticaria and angioedema in Korea," The Korean Journal of Internal Medicine, vol. 34, no. 2, pp. 418-425, 2019.

[9] J. E. Kim, Y. S. Eun, Y. M. Park et al., "Clinical characteristics of cholinergic urticaria in Korea," Annals of Dermatology, vol. 26, no. 2, pp. 189-194, 2014.

[10] T. Bito, Y. Sawada, and Y. Tokura, "Pathogenesis of cholinergic urticaria in relation to sweating," Allergology International, vol. 61, no. 4, pp. 539-544, 2012.

[11] J. V. Hirschmann, F. Lawlor, J. S. English, J. B. Louback, R. K. Winkelmann, and M. W. Greaves, "Cholinergic urticaria," Archives of Dermatology, vol. 123, no. 4, pp. 462-467, 1987.

[12] S. Takahagi, A. Tanaka, and M. Hide, "Sweat allergy," Allergology International, vol. 67, no. 4, pp. 435-441, 2018.

[13] M. Abajian, N. Schoepke, S. Altrichter, T. Zuberbier, and M. Maurer, "Physical urticarias and cholinergic urticaria," Immunology and Allergy Clinics of North America, vol. 34, no. 1, pp. 73-88, 2014.

[14] A. Kaplan, "Exercise-induced hives," Journal of Allergy and Clinical Immunology, vol. 73, no. 5, 5 Part 2, pp. 704-707, 1984.

[15] K. J. Collins, F. Sargent, and J. S. Weiner, "Excitation and depression of eccrine sweat glands by acetylcholine, acetyl- $\beta$ methylcholine and adrenaline," The Journal of Physiology, vol. 148, no. 3, pp. 592-614, 1959.

[16] J. A. Denburg, "Allergy and Allergic Diseases," in The New Mechanisms and Therapeutics, Humana Press Inc, New Jersey, 2nd edition, 1998.

[17] S. Nakamizo, G. Egawa, Y. Miyachi, and K. Kabashima, "Cholinergic urticaria: pathogenesis-based categorization and its treatment options," Journal of the European Academy of Dermatology and Venereology, vol. 26, no. 1, pp. 114-116, 2012.

[18] T. Horikawa, A. Fukunaga, and C. Nishigori, "New concepts of hive formation in cholinergic urticaria," Current Allergy and Asthma Reports, vol. 9, no. 4, pp. 273-279, 2009.

[19] J. Sánchez, E. Amaya, A. Acevedo, A. Celis, D. Caraballo, and R. Cardona, "Prevalence of inducible urticaria in patients with chronic spontaneous urticaria: associated risk factors," The Journal of Allergy and Clinical Immunology. In Practice, vol. 5, no. 2, pp. 464-470, 2017.

[20] K. Kulthanan, S. Jiamton, N. Thumpimukvatana, and S. Pinkaew, "Chronic idiopathic urticaria: prevalence and clinical course," The Journal of Dermatology, vol. 34, no. 5, pp. 294-301, 2007.

[21] N. Silpa-archa, K. Kulthanan, and S. Pinkaew, "Physical urticaria: prevalence, type and natural course in a tropical country," Journal of the European Academy of Dermatology and Venereology, vol. 25, no. 10, pp. 1194-1199, 2011.

[22] W. W. Duke, "Physical allergy as a cause of dermatoses," Archiv für Dermatologie und Syphilis, vol. 13, no. 2, pp. 176186, 1926.

[23] P. Vichyanond, S. Sunthornchart, V. Singhirannusorn, S. Ruangrat, S. Kaewsomboon, and N. Visitsunthorn, "Prevalence of asthma, allergic rhinitis and eczema among university students in Bangkok," Respiratory Medicine, vol. 96, no. 1, pp. 34-38, 2002.

[24] S. de Souza Campos Fernandes, C. R. de Andrade, C. G. Alvim, P. A. M. Camargos, and C. da Cunha Ibiapina, "Epidemiological trends of allergic diseases in adolescents," Jornal Brasileiro de Pneumologia, vol. 43, no. 5, pp. 368-372, 2017.

[25] C. E. Grattan, R. A. Sabroe, and M. W. Greaves, "Chronic urticaria," Journal of the American Academy of Dermatology, vol. 46, no. 5, pp. 645-660, 2002.

[26] A. M. Alsamarai, A. A. Hasan, and A. H. Alobaidi, "Evaluation of different combined regimens in the treatment of cholinergic urticaria," World Allergy Organization Journal, vol. 5, no. 8, pp. 88-93, 2012.

[27] G. Gastaminza, J. Azofra, J. M. Nunez-Cordoba et al., "Efficacy and safety of omalizumab (Xolair) for cholinergic urticaria in patients unresponsive to a double dose of antihistamines: a randomized mixed double-blind and open-label placebocontrolled clinical trial," The Journal of Allergy and Clinical Immunology: In Practice, vol. 7, no. 5, pp. 1599-609.e1, 2019.

[28] S. Joshi and D. A. Khan, "The expanding field of biologics in the management of chronic urticaria," The Journal of Allergy and Clinical Immunology: In Practice, vol. 5, no. 6, pp. 14891499, 2017.

[29] J. P. Arm, I. Bottoli, A. Skerjanec et al., "Pharmacokinetics, pharmacodynamics and safety of QGE031 (ligelizumab), a novel high-affinity anti-IgE antibody, in atopic subjects," Clinical and Experimental Allergy, vol. 44, no. 11, pp. 1371-1385, 2014.

[30] G. M. Gauvreau, J. M. Harris, L.-P. Boulet et al., "Targeting membrane-expressed IgE B cell receptor with an antibody to the M1 prime epitope reduces IgE production," Science Translational Medicine, vol. 6, no. 243, p. 243ra85, 2014. 